\title{
Determination of noise levels with respect to distance at selected workshops/factories in Itu Local Government Area of Akwa Ibom state, Nigeria
}

\author{
Menkiti, A. I ${ }^{1}$, Ekott, E. E. ${ }^{2}$ \\ ${ }^{1}$ Department of Physics, University of Calabar Calabar, Nigeria. \\ ${ }^{2}$ Department of Physics, University of Uyo, Nigeria.
}

\begin{abstract}
The measurement of noise levels with distances at selected workshops/factories in Itu Local Government Area of Akwa Ibom State, Nigeria was carried out. The noise levels measurements were made using a sound level meter, while distances were measured with a measuring tape. Firstly, the background noise levels (or noise levels without power generators and other noise makers from the workshops/factories) with distances were taken. Noise levels with power generators and other noise makers were then measured. Finally, the equivalent continuous noise levels, $L_{\text {eqs }}$ for them were calculated. The results of the survey show that the equivalent continuous noise level, $L_{e q}$ which is a measure of energy content of the noise decreases as the distance from the noise source increases, hence degree of bother (or annoyance) decreases with distance.
\end{abstract}

Keywords- Determination, distances, equivalent continuous noise levels, Itu Local Government Area, sound level meter.

\section{Introduction}

As with all pollutants, noise degrades the quality of our environment and is known to produce many adverse effects both on humans and structures. Noise has escalated to the point where it is now a major threat to the quality of our life. This increase in noise can be attributed to the ever increasing population of the world and the rising levels of economic affluence [1]. Noise is defined as unwanted sound, consequently it can be considered as the wrong sound in the wrong place at the wrong time. The degree of "unwantedness" is frequently a psychological matter since the effects of noise can range from moderate annoyance to permanent hearing loss, and may be rated differently by different observers. Therefore, it is often difficult to determine the benefits of reducing a specific noise. Noise does affect the inhabitants, humans, fauna, etc, in the natural environment. Although the impact of a particular noise source is limited to a specific area, noise is so pervasive that it is almost impossible to escape from it. Community Social surveys almost always rate noise among the most annoying environmental nuisances [2]. The rate at which environmental noise pollution is increasing in Itu Local Government Area of Akwa Ibom State, Nigeria needs a critical study. Environmental Protection Agency (EPA) of the United States of America recognised noise as a problem back in the 1970s. The EU Directive $(86 / 188 / \mathrm{EEC})$ is on the protection of workers from the risks related to exposure to noise at work. The objective of the directive is to reduce the level of noise experienced at work by taking action at the noise source. The EU directive specifies that when the daily exposure level exceeds $85 \mathrm{dBA}$, the worker is to be advised of the risks and trained to use ear protectors. If the daily exposure level exceeds $90 \mathrm{dBA}$, a programme to reduce levels should be put in place [2]. The British Columbia Work's Compensation Board (WCB) has set $85 \mathrm{~dB}$ as its maximum exposure limit in the work place. Above this level hearing protection should be worn. Its state that the threshold of pain is reached at $120 \mathrm{~dB}$ and it classifies $140 \mathrm{~dB}$ as extreme danger. World Health Organisation (WHO) values are similar while Environmental Protection Agency (EPA) tends to have even a stricter standard of $70 \mathrm{~dB}$ as a maximum safe noise level in work place. They gave the safe level around home to be $50-55 \mathrm{~dB}$. Studies have found that steady noise above $50 \mathrm{~dB}$ gives moderate annoyance and above $55 \mathrm{~dB}$ serious annoyance at home. For health and safety reasons in a non-work environment, $55 \mathrm{~dB}$ is set as a safety level for outside and $45 \mathrm{~dB}$ inside. Hospital and school safe levels are $35 \mathrm{~dB}$. Findings also show that the noise exposure limits in decibels for industrial workers in Nigeria are the same in dBA as that of the US Department of Labour as shown on Table 1.1 [3]. 
Table 1.1: Noise exposure limits for industrial workers in Nigeria

\begin{tabular}{ll}
\hline Exposure Time (h/day) & Permissible Exposure Limits in dB \\
\hline 8 & 90 \\
6 & 92 \\
4 & 95 \\
3 & 97 \\
2 & 100 \\
1.5 & 102 \\
1 & 105 \\
0.5 & 110 \\
0.25 or less & 115 \\
\hline
\end{tabular}

Studies show that excessive noise can cause hearing impairment, that certain levels and types of noise can cause heart attack, that body tissue resonances can be adversely affected by noise and that noise generally causes discomfort and annoyance to people exposed to it [4]. The hearing process consists of a number of separate processes. It should be noted that no simple and unique relationship exists between the physical measurement of sound and the human perception of the same sound [2]. The hearing damage potential of a given noise source depends not only on its level but also on its duration. It is generally accepted that a sound environment below $75 \mathrm{~dB}$ is not harmful (although much lower levels can cause annoyance and disturb sleep), while a single sound above $140 \mathrm{~dB}$ may produce permanent hearing damage. Between these two levels, the amount of hearing damage varies with the sound level, the length of exposure and the individual's susceptibility to noise. Other contributing factors are the number and length of quiet periods between exposures, the type of sound (continuous, intermittent or impulsive) and its frequency distribution. Sounds with most of their energy in the speech frequencies are more damaging. The EC Directive (86/188/EEC) on the protection of workers from the risks related to exposure to noise at work is incorporated into the laws of EC Member States [5]. It specifies that certain actions must be taken where the daily personal exposure (eight hour equivalent) of a worker to noise is likely to exceed $85 \mathrm{dBA}$ or where the maximum value of the unweighted instantaneous sound pressure is likely to be greater than $200 \mathrm{P}_{\mathrm{a}}$, equivalent to $140 \mathrm{~dB}$. In 1999, the World Health Organisation concluded that the available evidence shown suggested a weak associated between long term noise exposure above $67-70$ $\mathrm{dB}(\mathrm{A})$ and hypertension [6]. More recent studies have suggested that noise levels of $50 \mathrm{~dB}(\mathrm{~A})$ at night may also increase the risks of myocardial infarction by chronically elevating cortisol production [7]. According to Lesser W. Sontag of the Fels Research Institute (as presented in the pamphlet authored by the U.S Environmental Protection Agency in 1978); "there is ample evidence that environment has a role in shaping the physique, behaviour and function of animals including man from conception and not merely from birth. The foetus is capable of perceiving sounds and responding to them by motor activity and cardiac rate change". Noise exposure is deemed to be particularly pernicious when it occurs between 15 and 60 days after conception, when major internal organs and the central nervous system are formed. Later developmental effects occur as vasoconstriction in the mother reduces blood flow and hence oxygen and nutrition to the foetus. Low birth weights and noise were also associated with lower levels of certain hormone in the mother, these hormones being thought to affect foetal growth and to be a good indicator of protein production. The difference between the hormone levels of the pregnant mothers in noisy versus quiet areas increased as birth approaches. Children who live in noisy environments have been shown to have elevated blood pressures and elevated levels of stress induced hormones. Studies also suggest that when women are exposed to $76.5 \mathrm{~dB}$ aircraft noise, a small decrease in birth weight occurs [7]. Shouted conversations at the same distance are possible up to about $85 \mathrm{dBA}$. To permit normal conversations at distances of about five metres would require a background noise level below $50 \mathrm{dBA}$. Satisfactory telephone conversations need background levels less than about $80 \mathrm{dBA}$ [2]. High noise levels may reduce the accuracy of the work being undertaken rather than the quantity. Steady noises appear to have little effect on work performance unless the A-weighted noise level exceeds about $90 \mathrm{~dB}$ [8]. According to a WHO task group, daytime noise levels of less than $50 \mathrm{dBA}$ outdoors cause little or no serious annoyance in the community[9]. Intermittent or impulsive noises are particularly disturbing. Because of differences between people and locations, it is difficult to determine the noise level below which sleep interference will not occur [2]. Noise levels above $80 \mathrm{~dB}$ are associated with both an increase in aggressive behaviour and a decrease in behaviour helpful to others. The news media regularly report violent behaviour arising out of disputes over noise; in many cases these disputes end in injury or death [10].

\section{Materials and Methods}

Some workshops/factories and companies were identified around the place. Measurements of noise levels from them as they vary with distance were carried out. All the noise measurements were made using the sound level meter (SLM), model TES 1350A with $1 \frac{1}{2}$ inch electret condenser microphone. This model has both 
A and $\mathrm{C}$ weightings and $0.1 \mathrm{~dB}$ resolution with fast/slow response. It has high and low measuring ranges 35 to $100 \mathrm{~dB}$ and 65 to $160 \mathrm{~dB}$ respectively. Measurements were taken by setting the sound level meter to Aweighting network in all the sampling locations. The wind speed and direction relation to the microphone was considered. This is because at higher wind speed (i.e above $5 \mathrm{~m} / \mathrm{s}$ ) turbulent noise caused by the wind may mask the noise source being measured. This implies that valid measurements can be taken in wind speed up to $5 \mathrm{~m} / \mathrm{s}$. Measurement may be acceptable with wind speeds up to $10 \mathrm{~m} / \mathrm{s}$. In general the peaks of wind noise should be at least $10 \mathrm{~dB}$ below the noise source being measured [2]. Hence, during sound level measurements, windshield was always used (i.e. for outdoor measurements). Slow response was used for comparatively stable noise measurement. For instance, work place noise level measurements were taken on slow response. Here, the response rate is the time period over which the instrument averages the sound level before displaying it on the readout. Fast response was used for fast varying noise. Measurements of workplace sound pressure were made in the undisturbed sound field in the workplace, with the microphone located at the position normally occupied by the ear exposed to the highest value of exposure [11]. All noise level measurements were made using the sound level meter, while distance measurements were made using a measuring tape. Finally, the equivalent continuous noise levels, $\mathrm{L}_{\mathrm{eqs}}$ for them at a particular distance, $d$ were calculated using the formula [2]:

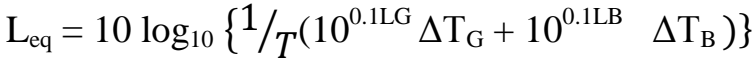

where, $\quad T=5$ minutes; $\Delta \mathrm{T}_{\mathrm{G}}=2$ minutes $; \Delta \mathrm{T}_{\mathrm{B}}=3$ minutes; $\mathrm{L}_{\mathrm{G}}=$ Generator noise level in $\mathrm{dBA}$

$\mathrm{L}_{\mathrm{B}}=$ Background noise level in $\mathrm{dBA}$.

III. Results and Discussion

3.1 Akwa Ibom Broadcasting Corporation Transmitting Station, Ntak Inyang

Table 3.1: Noise levels and distance measurements from a 300 kVA Perkins power generator (2010)

\begin{tabular}{llllll}
\hline $\begin{array}{l}\text { Distance, } \\
(\mathbf{m})\end{array}$ & $\begin{array}{l}\text { Background } \\
\text { Noise, } \mathbf{L}_{\mathbf{B}}(\mathbf{d B A})\end{array}$ & $\begin{array}{l}\text { Noise Level } \\
\text { Generator, } \\
(\mathbf{d B A})\end{array}$ & $\begin{array}{r}\text { with } \\
\mathbf{L}\end{array}$ & $\begin{array}{l}\text { Generator Noise } \\
\text { Level, } \mathbf{L}_{\mathbf{G}}(\mathbf{d B A})\end{array}$ & $\begin{array}{l}\text { Equivalent } \\
\text { Continuous } \\
\text { Level, Leq (dBA) }\end{array}$ \\
\hline $\mathbf{5}$ & 36.0 & 92.0 & 56.0 & $\mathbf{5 2 . 1}$ \\
$\mathbf{1 0}$ & 37.0 & 86.3 & 49.3 & $\mathbf{4 5 . 7}$ \\
$\mathbf{1 5}$ & 36.9 & 81.3 & 44.4 & $\mathbf{4 1 . 4}$ \\
$\mathbf{2 0}$ & 39.0 & 75.7 & 36.7 & $\mathbf{3 8 . 2}$ \\
$\mathbf{2 5}$ & 36.7 & 73.6 & 36.9 & $\mathbf{3 6 . 8}$ \\
$\mathbf{3 0}$ & 35.5 & 72.3 & 36.8 & $\mathbf{3 6 . 1}$ \\
$\mathbf{3 5}$ & 35.9 & 70.6 & 34.7 & $\mathbf{3 5 . 5}$ \\
$\mathbf{4 0}$ & 36.1 & 68.9 & 32.8 & $\mathbf{3 5 . 1}$ \\
$\mathbf{4 5}$ & 37.9 & 65.8 & 27.9 & $\mathbf{3 6 . 0}$ \\
$\mathbf{5 0}$ & 40.0 & 63.9 & 23.9 & $\mathbf{3 7 . 9}$ \\
$\mathbf{5 5}$ & 40.2 & 62.5 & 22.3 & $\mathbf{3 8 . 0}$ \\
$\mathbf{6 0}$ & 40.1 & 60.7 & 20.6 & $\mathbf{3 7 . 9}$ \\
$\mathbf{6 5}$ & 40.4 & 59.8 & 15.4 & $\mathbf{3 8 . 2}$ \\
$\mathbf{7 0}$ & 40.0 & 58.8 & 18.8 & $\mathbf{3 7 . 8}$ \\
$\mathbf{7 5}$ & 38.2 & 58.1 & 19.9 & $\mathbf{3 6 . 0}$ \\
$\mathbf{8 0}$ & 39.0 & 56.7 & 17.7 & $\mathbf{3 6 . 8}$ \\
\hline $\mathbf{8 5}$ & 39.5 & 55.9 & 16.4 & $\mathbf{3 7 . 3}$ \\
\hline $\mathbf{9 0}$ & 38.3 & 53.2 & 14.9 & $\mathbf{3 6 . 1}$ \\
\hline $\mathbf{9 5}$ & 37.6 & 48.6 & 11.0 & $\mathbf{3 5 . 4}$ \\
\hline $\mathbf{1 0 0}$ & $\mathbf{3 5 . 2}$ & $\mathbf{5 0 . 9}$ & $\mathbf{1 5 . 7}$ & $\mathbf{3 3 . 0}$ \\
\hline
\end{tabular}




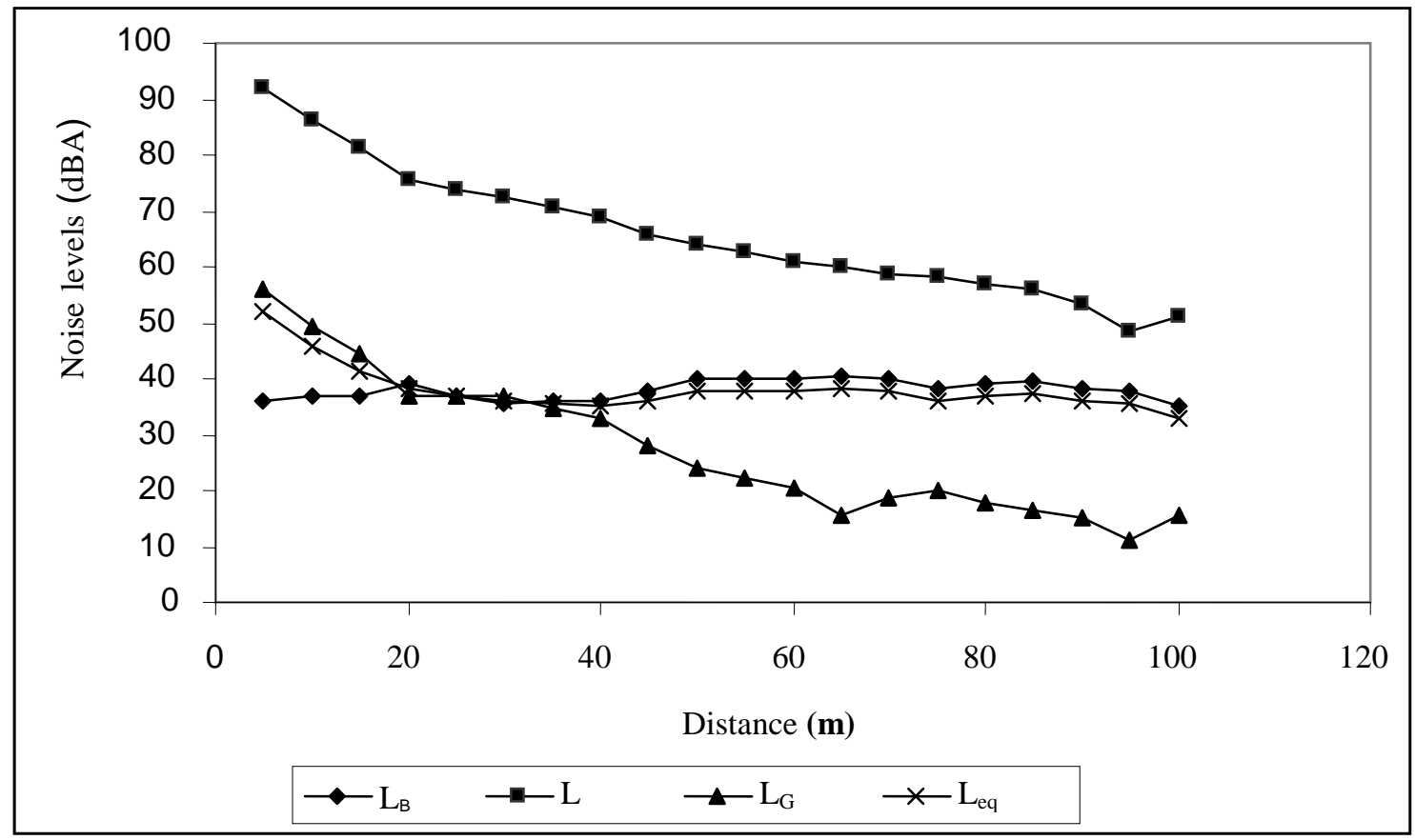

Figure 3.1: A plot of a $300 \mathrm{kVA}$ Perkins power generator noise levels against distance

Table 3.2: Noise levels and distance measurements from a 375 kVA Perkins power generator (2010)

\begin{tabular}{|c|c|c|c|c|}
\hline $\begin{array}{l}\text { Distance, } \\
\text { d (m) }\end{array}$ & $\begin{array}{l}\text { Background } \\
\text { Noise, } L_{B}(d B A)\end{array}$ & $\begin{array}{l}\text { Noise Level with } \\
\text { Generator, L } \\
\text { (dBA) }\end{array}$ & $\begin{array}{l}\text { Generator } \\
\text { Noise Level, } \\
\text { L }_{G}(\text { dBA })\end{array}$ & $\begin{array}{l}\text { Equivalent } \\
\text { Continuous Noise } \\
\text { Level, Leq (dBA) }\end{array}$ \\
\hline 5 & 35.9 & 96.6 & 60.7 & 56.7 \\
\hline 10 & 35.5 & 90.9 & 55.4 & 51.5 \\
\hline 15 & 35.6 & 86.8 & 51.2 & 47.4 \\
\hline 20 & 36.2 & 82.9 & 46.7 & 39.7 \\
\hline 25 & 37.0 & 79.3 & 42.3 & 39.9 \\
\hline 30 & 36.9 & 75.7 & 38.8 & 37.8 \\
\hline 35 & 35.8 & 70.4 & 34.6 & 35.4 \\
\hline 40 & 36.0 & 68.9 & 32.9 & 35.0 \\
\hline 45 & 36.9 & 64.5 & 27.6 & 35.0 \\
\hline 50 & 38.6 & 61.1 & 22.5 & 36.5 \\
\hline 55 & 39.0 & 62.0 & 23.0 & 36.9 \\
\hline 60 & 39.9 & 60.7 & 20.8 & 37.7 \\
\hline 65 & 40.2 & 58.8 & 18.6 & 38.0 \\
\hline 70 & 39.3 & 55.0 & 15.7 & 37.1 \\
\hline 75 & 39.6 & 53.8 & 14.2 & 37.4 \\
\hline 80 & 40.7 & 59.0 & 18.3 & 38.5 \\
\hline 85 & 39.0 & 57.1 & 18.1 & 36.8 \\
\hline 90 & 36.8 & 54.0 & 17.2 & 34.6 \\
\hline 95 & 38.3 & 50.9 & 12.6 & 36.1 \\
\hline 100 & 39.1 & 48.8 & 9.8 & 36.9 \\
\hline
\end{tabular}




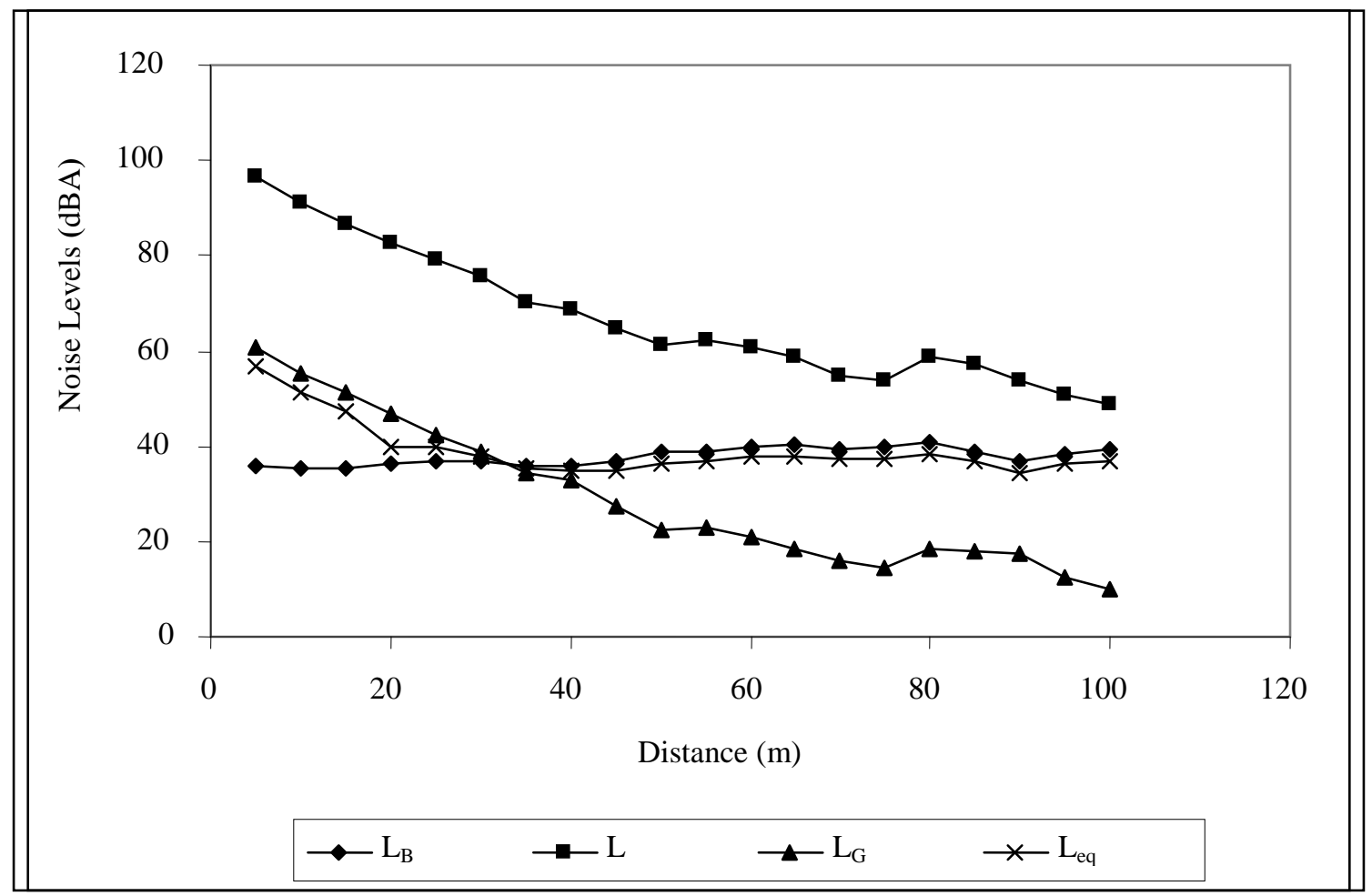

Figure 3.2: A plot of a $375 \mathrm{kVA}$ Perkins power generator noise levels again distance

Table 3.3: Noise levels and distance measurements from a $500 \mathrm{kVA}$ Perkins power generator (2010)

\begin{tabular}{llllll}
\hline $\begin{array}{l}\text { Distance, } \\
(\mathbf{m})\end{array}$ & $\begin{array}{l}\text { Background Noise, } \\
\mathbf{L}_{\mathbf{B}}(\mathbf{d B A})\end{array}$ & $\begin{array}{l}\text { Noise } \\
\text { Generator, } \mathbf{L}(\mathbf{d B A})\end{array}$ & $\begin{array}{l}\text { Generator } \\
\text { Level, } \mathbf{L}_{\mathbf{G}}(\mathbf{d B A})\end{array}$ & $\begin{array}{l}\text { Equivalent } \\
\text { Continuous } \\
\text { Level, Leq }(\mathbf{d B A})\end{array}$ \\
\hline $\mathbf{5}$ & 35.6 & 99.6 & 64.0 & $\mathbf{6 0 . 0}$ \\
$\mathbf{1 0}$ & 35.4 & 92.1 & 56.7 & $\mathbf{5 2 . 8}$ \\
$\mathbf{1 5}$ & 36.0 & 87.6 & 51.6 & $\mathbf{4 7 . 8}$ \\
$\mathbf{2 0}$ & 36.5 & 83.2 & 46.7 & $\mathbf{4 3 . 3}$ \\
$\mathbf{2 5}$ & 40.3 & 76.0 & 35.7 & $\mathbf{3 9 . 0}$ \\
$\mathbf{3 0}$ & 40.0 & 74.8 & 34.8 & $\mathbf{3 8 . 6}$ \\
$\mathbf{3 5}$ & 38.1 & 72.7 & 34.6 & $\mathbf{3 7 . 0}$ \\
$\mathbf{4 0}$ & 36.2 & 71.6 & 35.4 & $\mathbf{3 5 . 9}$ \\
$\mathbf{4 5}$ & 36.0 & 69.9 & 33.9 & $\mathbf{3 5 . 3}$ \\
$\mathbf{5 0}$ & 36.2 & 68.0 & 31.8 & $\mathbf{3 4 . 9}$ \\
$\mathbf{5 5}$ & 36.0 & 66.0 & 30.0 & $\mathbf{3 4 . 4}$ \\
$\mathbf{6 0}$ & 35.0 & 65.3 & 30.3 & $\mathbf{3 3 . 7}$ \\
$\mathbf{6 5}$ & 35.1 & 64.0 & 28.9 & $\mathbf{3 5 . 5}$ \\
$\mathbf{7 0}$ & 41.1 & 62.6 & 21.5 & $\mathbf{3 8 . 9}$ \\
$\mathbf{7 5}$ & 38.2 & 61.8 & 23.6 & $\mathbf{3 6 . 1}$ \\
$\mathbf{8 0}$ & 37.6 & 60.0 & 22.4 & $\mathbf{3 5 . 5}$ \\
$\mathbf{8 5}$ & 38.0 & 58.0 & 20.0 & $\mathbf{3 5 . 8}$ \\
$\mathbf{9 0}$ & 39.6 & 56.7 & 17.1 & $\mathbf{3 7 . 4}$ \\
\hline $\mathbf{9 5}$ & 39.1 & 55.0 & 15.9 & $\mathbf{3 7 . 1}$ \\
\hline $\mathbf{1 0 0}$ & $\mathbf{4 0 . 2}$ & $\mathbf{5 2 . 2}$ & & $\mathbf{3 8 . 0}$ \\
\hline
\end{tabular}




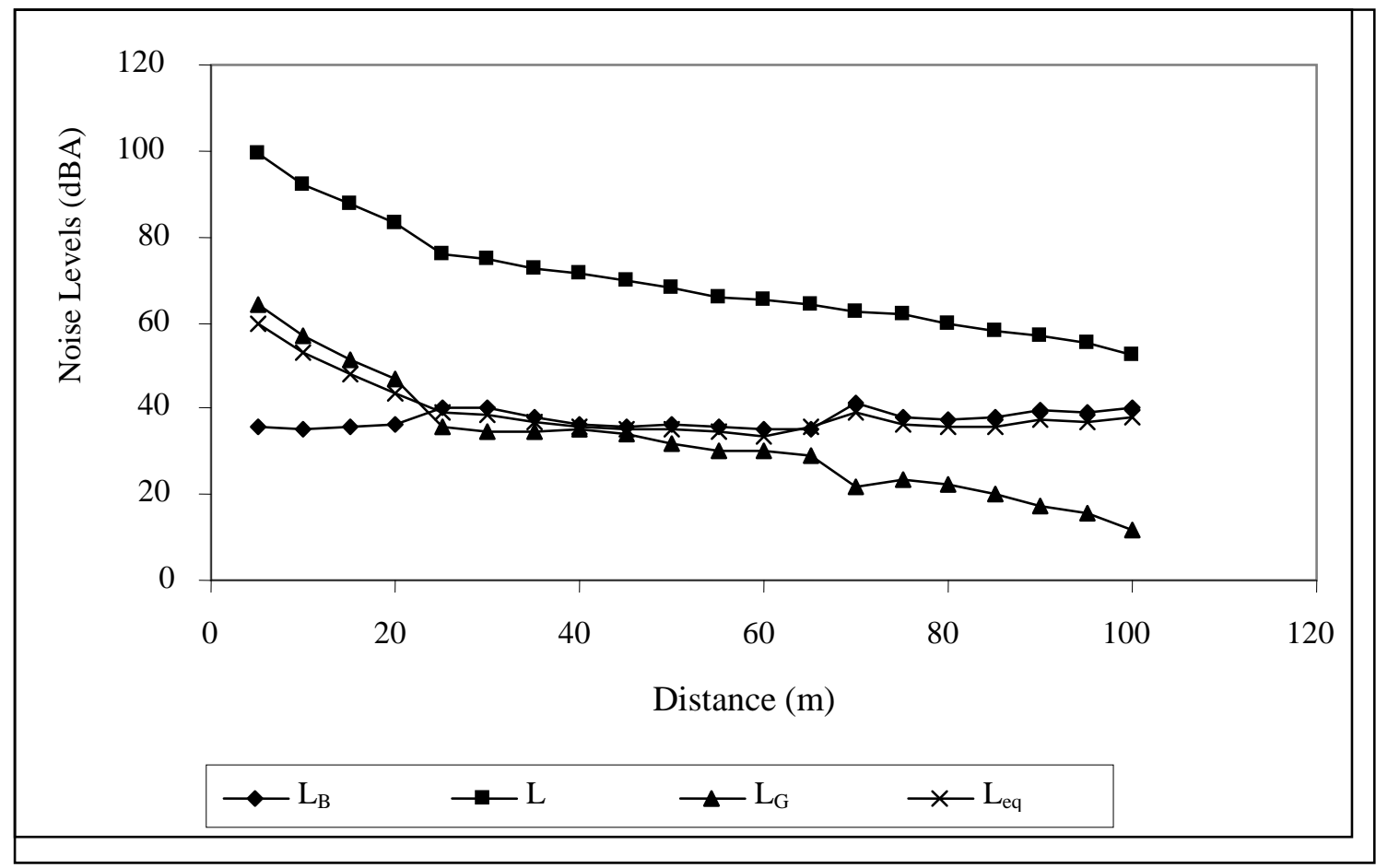

Figure 3.3: A plot of a $500 \mathrm{kVA}$ Perkins power generator noise levels against distance

\subsection{UPTECH Metal Construction Works, 180 Calabar-Itu Express Way}

Table 3.4: Noise levels and distance measurements from a $100 \mathrm{~kW}$ Sifang power generator and other noise makers in UPTECH metal construction works (2010)

\begin{tabular}{|c|c|c|c|c|}
\hline $\begin{array}{l}\text { Distance, } \mathrm{d} \\
\text { (m) }\end{array}$ & $\begin{array}{l}\text { Background Noise, } \\
\mathrm{L}_{\mathrm{B}}(\mathrm{dBA})\end{array}$ & $\begin{array}{l}\text { Noise Level with } \\
\text { Generator, L (dBA) }\end{array}$ & 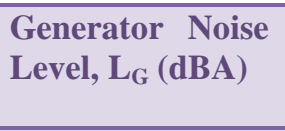 & $\begin{array}{l}\text { Equivalent } \\
\text { Continuous Noise } \\
\text { Level, Leq (dBA) }\end{array}$ \\
\hline 5 & 35.9 & 89.7 & 53.8 & 49.9 \\
\hline 10 & 36.2 & 86.2 & 50.0 & 46.3 \\
\hline 15 & 36.0 & 82.8 & 46.8 & 43.3 \\
\hline 20 & 36.8 & 78.9 & 42.1 & 39.7 \\
\hline 25 & 36.1 & 74.7 & 38.6 & 37.3 \\
\hline 30 & 38.0 & 70.6 & 32.6 & 36.5 \\
\hline 35 & 37.8 & 67.5 & 29.7 & 36.0 \\
\hline 40 & 40.2 & 66.7 & 26.8 & 38.1 \\
\hline 45 & 38.5 & 64.9 & 26.4 & 36.5 \\
\hline 50 & 37.6 & 61.3 & 23.7 & 35.5 \\
\hline 55 & 38.0 & 58.4 & 20.4 & 35.8 \\
\hline 60 & 37.8 & 55.5 & 17.7 & 35.6 \\
\hline 65 & 38.1 & 52.8 & 14.7 & 35.9 \\
\hline 70 & 39.0 & 49.1 & 10.1 & 36.8 \\
\hline 75 & 36.6 & 48.8 & 12.2 & 34.4 \\
\hline 80 & 35.8 & 43.6 & 7.8 & 33.6 \\
\hline 85 & 35.0 & 41.3 & 6.3 & 32.8 \\
\hline 90 & 36.1 & 44.9 & 8.8 & 33.9 \\
\hline 95 & 36.3 & 42.2 & 5.9 & 34.1 \\
\hline 100 & 36.0 & 39.9 & 3.9 & 33.8 \\
\hline
\end{tabular}




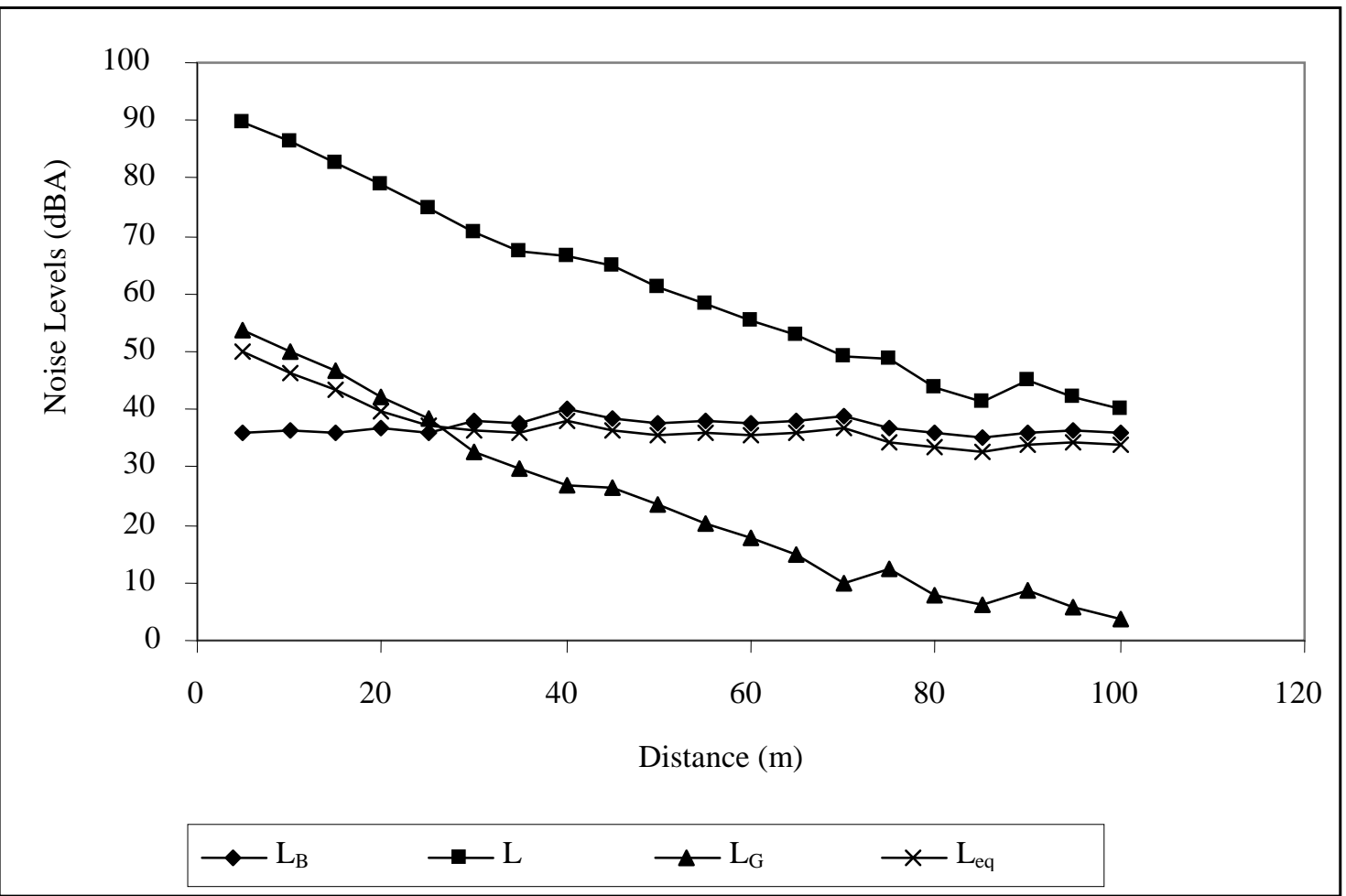

Figure 3.4: A plot of noise levels against distance for a $100 \mathrm{~kW}$ Sifang power generator and other noise makers in UPTECH metal construction works

Table 3.5: Noise levels and distance measurements from another Sifang power generator and other noise makers in UPTECH metal construction works (2010)

\begin{tabular}{llllll}
\hline $\begin{array}{l}\text { Distance, } \\
(\mathbf{m})\end{array}$ & $\begin{array}{l}\text { Background Noise, } \\
\mathbf{L}_{\mathbf{B}}(\mathbf{d B A})\end{array}$ & $\begin{array}{l}\text { Noise } \\
\text { Generator, } \mathbf{L}(\mathbf{d B A})\end{array}$ & $\begin{array}{l}\text { Generator } \\
\text { Level, } \mathbf{L}_{\mathrm{G}}(\mathbf{d B A})\end{array}$ & $\begin{array}{l}\text { Equivalent } \\
\text { Continuous } \\
\text { Level, Leq (dBA) }\end{array}$ \\
\hline $\mathbf{5}$ & 36.5 & 84.6 & 48.1 & $\mathbf{4 4 . 5}$ \\
$\mathbf{1 0}$ & 35.5 & 80.1 & 44.6 & $\mathbf{4 1 . 6}$ \\
$\mathbf{1 5}$ & 36.9 & 78.9 & 42.0 & $\mathbf{3 9 . 7}$ \\
$\mathbf{2 0}$ & 40.0 & 76.0 & 36.0 & $\mathbf{3 8 . 8}$ \\
$\mathbf{2 5}$ & 38.7 & 73.6 & 34.9 & $\mathbf{3 7 . 5}$ \\
$\mathbf{3 0}$ & 36.3 & 70.4 & 33.1 & $\mathbf{3 5 . 3}$ \\
$\mathbf{3 5}$ & 35.5 & 67.8 & 32.3 & $\mathbf{3 4 . 5}$ \\
$\mathbf{4 0}$ & 35.7 & 64.3 & 28.6 & $\mathbf{3 4 . 0}$ \\
$\mathbf{4 5}$ & 35.9 & 61.9 & 26.0 & $\mathbf{3 4 . 0}$ \\
$\mathbf{5 0}$ & 35.5 & 57.2 & 21.7 & $\mathbf{3 3 . 4}$ \\
$\mathbf{5 5}$ & 36.0 & 57.4 & 21.4 & $\mathbf{3 3 . 9}$ \\
$\mathbf{6 0}$ & 35.9 & 56.7 & 20.8 & $\mathbf{3 3 . 8}$ \\
$\mathbf{6 5}$ & 36.3 & 54.9 & 18.6 & $\mathbf{3 4 . 6}$ \\
$\mathbf{7 0}$ & 36.8 & 53.8 & 17.0 & $\mathbf{3 4 . 6}$ \\
$\mathbf{7 5}$ & 40.0 & 55.9 & 15.9 & $\mathbf{3 7 . 8}$ \\
$\mathbf{8 0}$ & 41.2 & 54.9 & 13.2 & $\mathbf{3 9 . 0}$ \\
$\mathbf{8 5}$ & 39.1 & 47.9 & 8.8 & $\mathbf{3 6 . 9}$ \\
$\mathbf{9 0}$ & 38.7 & 47.0 & 8.3 & $\mathbf{3 6 . 5}$ \\
$\mathbf{9 5}$ & 38.9 & 43.9 & 5.0 & $\mathbf{3 6 . 7}$ \\
\hline $\mathbf{1 0 0}$ & $\mathbf{4 2 . 3}$ & $\mathbf{4 3 . 6}$ & $\mathbf{1 . 3}$ & $\mathbf{4 0 . 1}$ \\
\hline
\end{tabular}




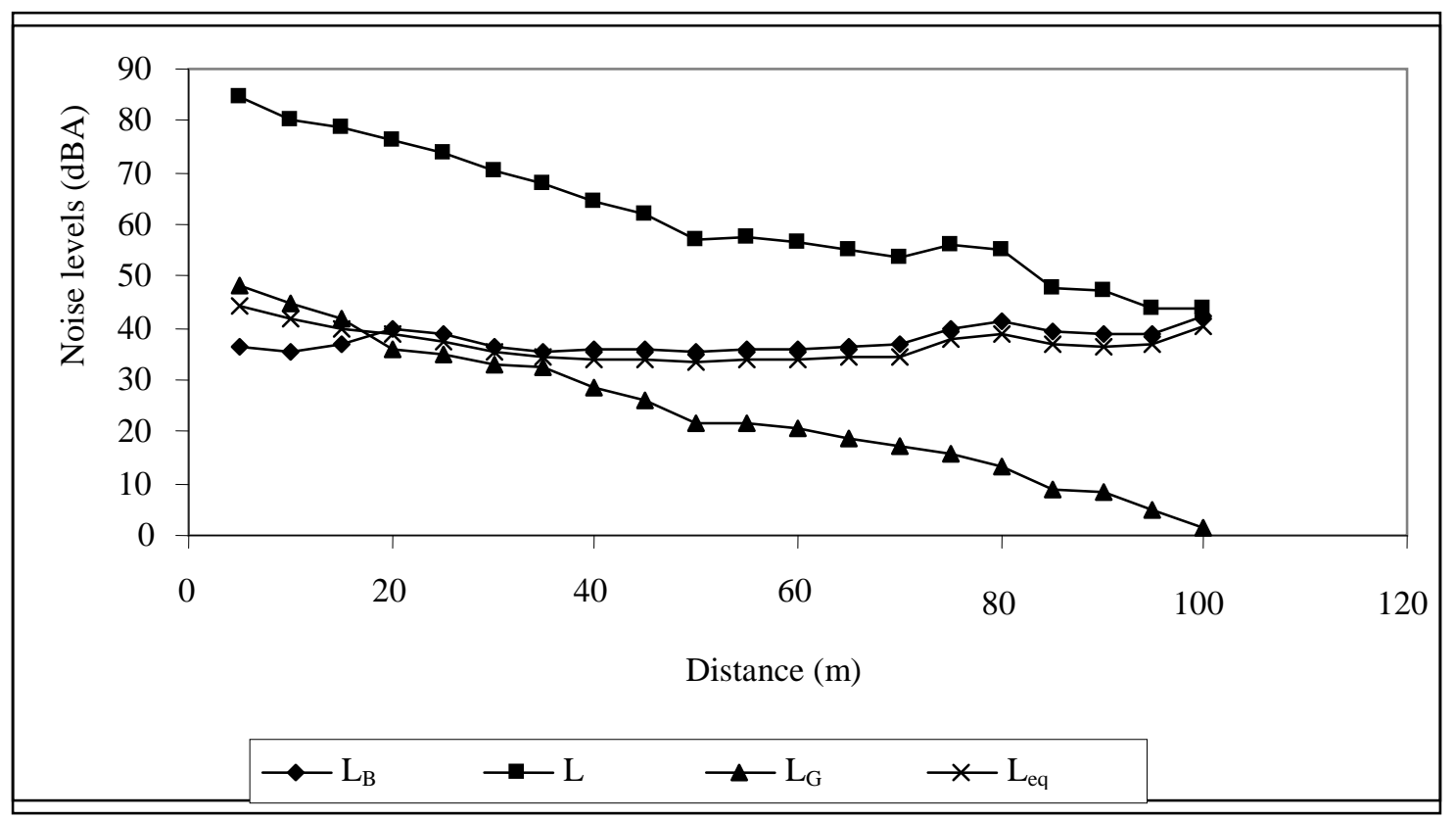

Figure 3.5: A plot of noise levels against distance for another Sifang power generator and other noise makers in UPTECH metal construction works

Table 3.6: Noise levels and distance measurements from three Sifang power generators and other noise makers in UPTECH metal construction works (2010)

\begin{tabular}{lllll}
\hline $\begin{array}{l}\text { Distance, } \\
(\mathbf{m})\end{array}$ & $\begin{array}{l}\text { Background Noise, } \\
\mathbf{L}_{\mathbf{B}}(\mathbf{d B A})\end{array}$ & $\begin{array}{l}\text { Noise } \\
\text { Generator, } \mathbf{L}(\mathbf{d B A})\end{array}$ & $\begin{array}{l}\text { Generator Noise } \\
\text { Level, } \mathbf{L}_{\mathrm{G}}(\mathbf{d B A})\end{array}$ & $\begin{array}{l}\text { Equivalent } \\
\text { Continuous } \\
\text { Level, Leq (dBA) }\end{array}$ \\
\hline $\mathbf{5}$ & 36.8 & 118.0 & 81.2 & $\mathbf{7 7 . 2}$ \\
$\mathbf{1 0}$ & 36.9 & 110.6 & 73.7 & $\mathbf{6 9 . 7}$ \\
$\mathbf{1 5}$ & 36.0 & 101.9 & 65.9 & $\mathbf{6 1 . 9}$ \\
$\mathbf{2 0}$ & 35.7 & 94.3 & 58.6 & $\mathbf{5 4 . 7}$ \\
$\mathbf{2 5}$ & 35.5 & 91.5 & 56.0 & $\mathbf{5 2 . 1}$ \\
$\mathbf{3 0}$ & 36.3 & 86.7 & 50.4 & $\mathbf{4 6 . 7}$ \\
$\mathbf{3 5}$ & 37.4 & 84.7 & 47.3 & $\mathbf{4 3 . 9}$ \\
$\mathbf{4 0}$ & 38.5 & 83.0 & 44.5 & $\mathbf{4 1 . 9}$ \\
$\mathbf{4 5}$ & 38.0 & 78.9 & 40.9 & $\mathbf{3 9 . 4}$ \\
$\mathbf{5 0}$ & 38.8 & 76.8 & 38.0 & $\mathbf{3 8 . 5}$ \\
$\mathbf{5 5}$ & 38.5 & 75.0 & 36.5 & $\mathbf{3 7 . 8}$ \\
$\mathbf{6 0}$ & 39.2 & 72.8 & 33.6 & $\mathbf{3 7 . 7}$ \\
$\mathbf{6 5}$ & 38.9 & 68.1 & 29.2 & $\mathbf{3 7 . 0}$ \\
$\mathbf{7 0}$ & 37.0 & 61.9 & 24.9 & $\mathbf{3 5 . 0}$ \\
$\mathbf{7 5}$ & 40.0 & 63.4 & 23.4 & $\mathbf{3 7 . 8}$ \\
$\mathbf{8 0}$ & 36.9 & 60.8 & 23.9 & $\mathbf{3 4 . 8}$ \\
\hline $\mathbf{8 5}$ & 39.6 & 63.3 & 23.7 & $\mathbf{3 7 . 5}$ \\
$\mathbf{9 0}$ & 43.5 & 62.8 & 19.3 & $\mathbf{4 1 . 3}$ \\
$\mathbf{9 5}$ & 45.3 & 58.1 & 12.8 & $\mathbf{4 3 . 1}$ \\
\hline $\mathbf{1 0 0}$ & $\mathbf{4 5 . 1}$ & $\mathbf{5 9 . 9}$ & $\mathbf{1 4 . 8}$ & $\mathbf{4 2 . 9}$ \\
\hline
\end{tabular}




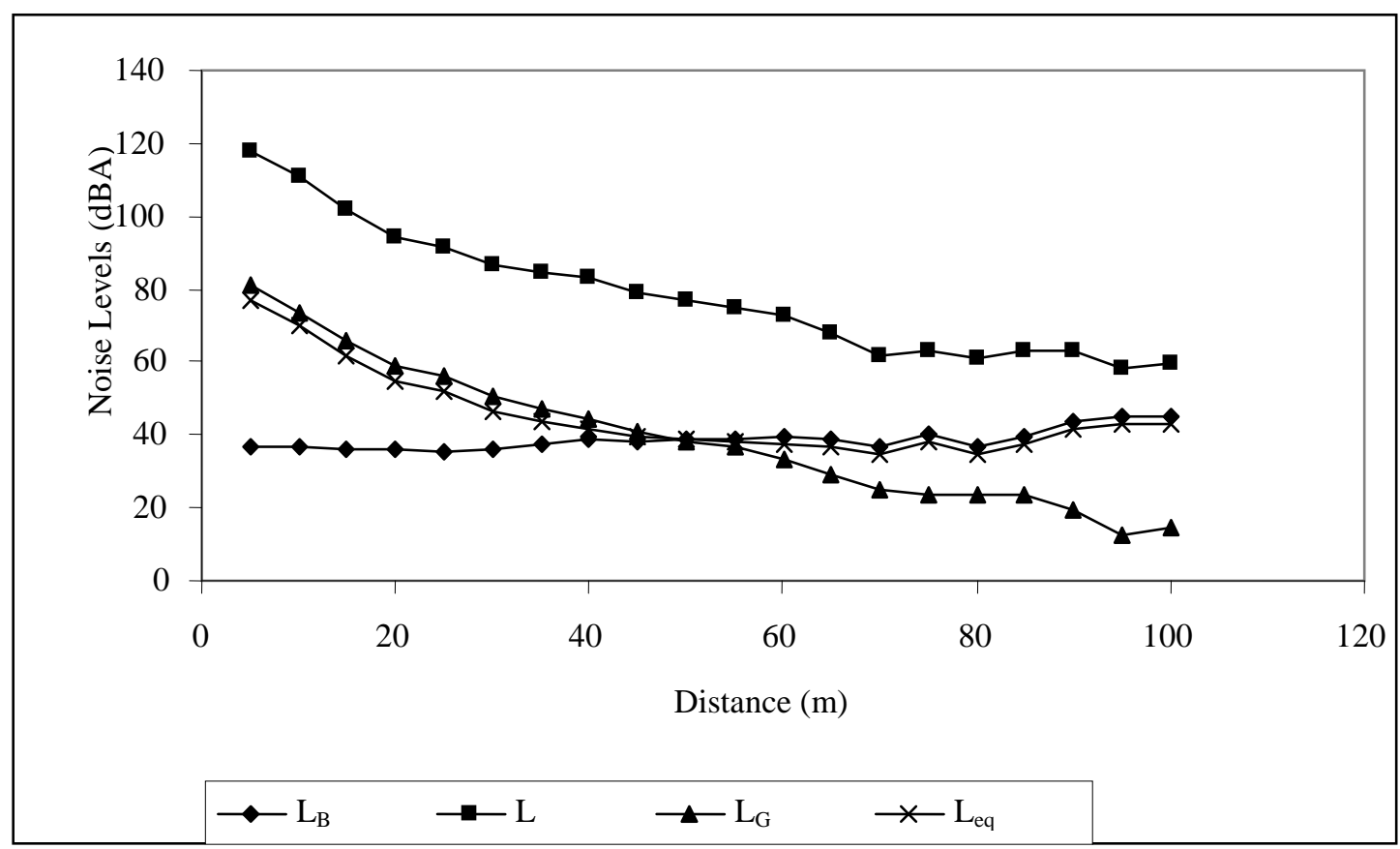

Figure 3.6: A plot of noise levels against distance for three Sifang power generators and other noise makers in UPTECH metal construction works

\subsection{Holy Saviour Water Company, Ikot Ekwere Itam}

Table 3.7: Noise levels with distances from Holy Saviour water company (2010)

\begin{tabular}{llllll}
\hline $\begin{array}{l}\text { Distance, } \\
\mathbf{d}(\mathbf{m})\end{array}$ & $\begin{array}{l}\text { Background } \\
\text { Noise, } \mathbf{L}_{\mathbf{B}}(\mathbf{d B A})\end{array}$ & $\begin{array}{l}\text { Noise } \\
\text { Generator, } \\
(\mathbf{d B A})\end{array}$ & $\begin{array}{r}\text { with } \\
\mathbf{L}\end{array}$ & $\begin{array}{l}\text { Generator } \\
\text { Level, } \mathbf{L}_{\mathrm{G}}(\mathbf{d B A})\end{array}$ & $\begin{array}{l}\text { Equivalent } \\
\text { Continuous } \\
\text { Level, Leq (dBA) }\end{array}$ \\
\hline $\mathbf{5}$ & 38.2 & 76.9 & 38.7 & $\mathbf{3 8 . 4}$ \\
$\mathbf{1 0}$ & 36.5 & 74.6 & 38.1 & $\mathbf{3 7 . 2}$ \\
$\mathbf{1 5}$ & 35.8 & 70.8 & 35.0 & $\mathbf{3 5 . 5}$ \\
$\mathbf{2 0}$ & 37.0 & 66.3 & 29.3 & $\mathbf{3 5 . 2}$ \\
$\mathbf{2 5}$ & 37.1 & 63.7 & 26.6 & $\mathbf{3 0 . 8}$ \\
$\mathbf{3 0}$ & 36.9 & 59.2 & 22.3 & $\mathbf{3 4 . 8}$ \\
$\mathbf{3 5}$ & 38.4 & 57.5 & 19.1 & $\mathbf{3 6 . 2}$ \\
$\mathbf{4 0}$ & 41.0 & 55.0 & 14.0 & $\mathbf{3 8 . 8}$ \\
$\mathbf{4 5}$ & 42.3 & 52.0 & 9.7 & $\mathbf{4 0 . 1}$ \\
$\mathbf{5 0}$ & 40.8 & 50.7 & 9.9 & $\mathbf{3 8 . 6}$ \\
$\mathbf{5 5}$ & 39.7 & 51.4 & 11.7 & $\mathbf{3 7 . 5}$ \\
$\mathbf{6 0}$ & 44.2 & 55.3 & 11.1 & $\mathbf{4 2 . 0}$ \\
$\mathbf{6 5}$ & 51.6 & 57.1 & 5.5 & $\mathbf{4 9 . 4}$ \\
$\mathbf{7 0}$ & 55.0 & 55.0 & 0.0 & $\mathbf{5 2 . 8}$ \\
$\mathbf{7 5}$ & 49.9 & 54.7 & 4.8 & $\mathbf{4 7 . 7}$ \\
$\mathbf{8 0}$ & 49.1 & 53.6 & 4.5 & $\mathbf{4 6 . 9}$ \\
$\mathbf{8 5}$ & 49.8 & 55.6 & 5.8 & $\mathbf{4 7 . 6}$ \\
$\mathbf{9 0}$ & 51.3 & 56.0 & 4.7 & $\mathbf{4 9 . 1}$ \\
$\mathbf{9 5}$ & 52.2 & 55.8 & 3.6 & $\mathbf{5 0 . 0}$ \\
\hline $\mathbf{1 0 0}$ & $\mathbf{5 3 . 8}$ & $\mathbf{5 5 . 6}$ & $\mathbf{1 . 8}$ & $\mathbf{5 1 . 6}$ \\
\hline
\end{tabular}




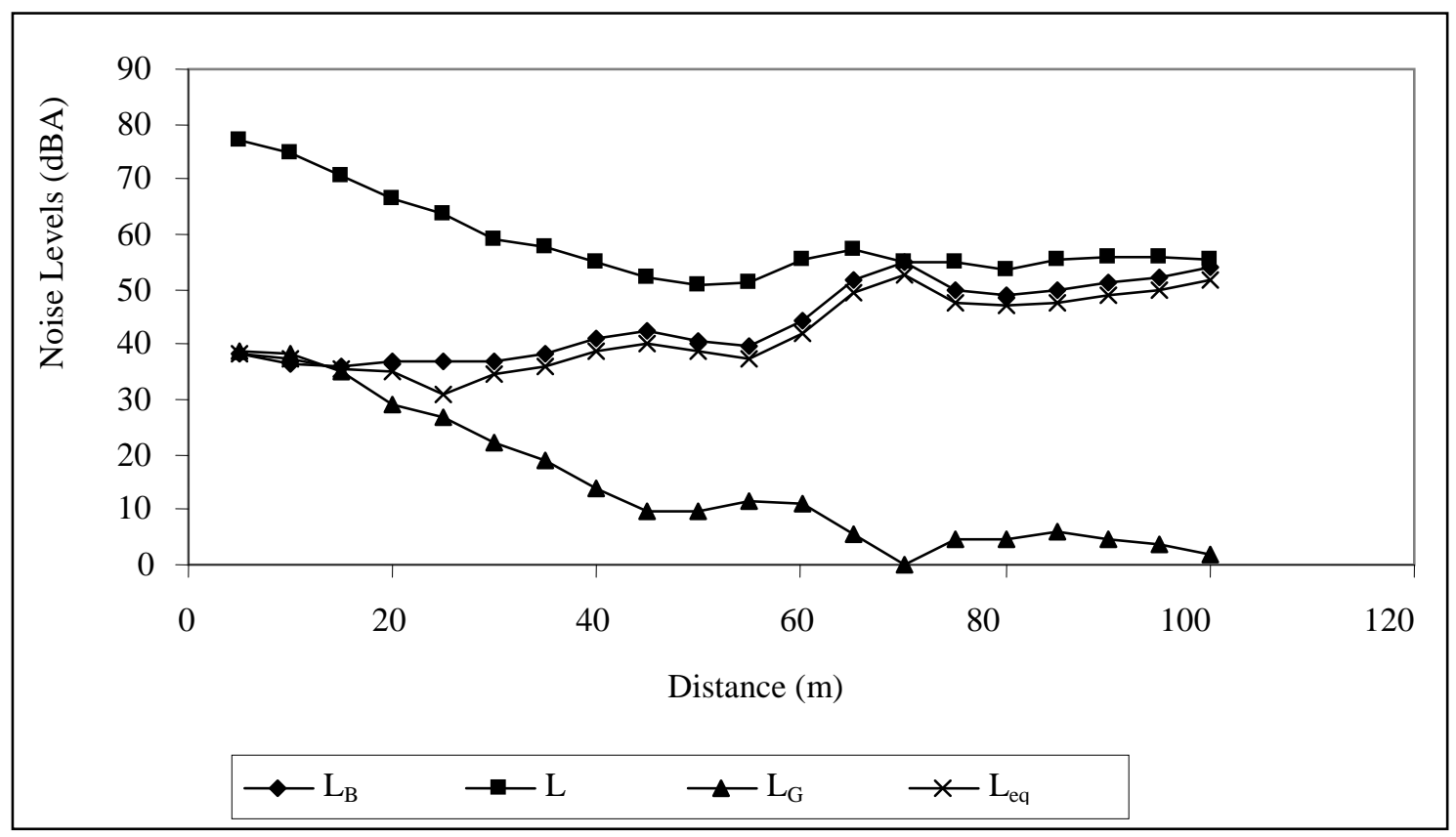

Figure 3.7: A plot of noise levels against distance in Holy Saviour water company

\section{Discussion}

The results of the survey (Table 3.1 and Fig. 3.1) show that when the power generator is switched off, the area is conducive for both the workers and the residents. But when the generator is in use, noise emitted affects the people up to a distance of 80 metres away from it. At a distance of 5 metres from the generator, a total noise level of $92.0 \mathrm{dBA}$ is recorded. This level $(92.0 \mathrm{dBA})$ is above the World Health Organization (WHO) standard of $85 \mathrm{~dB}$ in a work place. Hence, the workers should be advised to use ear protector and they should not be exposed to it beyond duration of 8 hours per day as stated in Table 1.1. The adverse effects of the generator noise become more prominent at areas where it level and the equivalent continuous level are greater than the background noise level. At a distance of 85 metres away from the generator, a total noise level of 55.9 $\mathrm{dBA}$ is recorded. This level $(55.9 \mathrm{dBA})$ is $0.9 \mathrm{dBA}$ above the WHO standard level of $55 \mathrm{~dB}$ for a non work environment. It has a generator noise level of $16.4 \mathrm{dBA}$ and an equivalent continuous level of $37.3 \mathrm{dBA}$. From about a distance of 90 metres away, the residents do not have a generator as their major source of noise. Table 3.1 and Fig. 3.1 also show that the total noise level, the generator noise level and the equivalent continuous level fall as distance increases. Table 3.2 and Fig. 3.2 show that a sound wave from a source is attenuated or decreased with distance. With this generator in use, its noise affects the residents up to a distance of 85 metres away from it. At this distance ( 85 metres), the total noise level is $2.1 \mathrm{dBA}$ above the WHO standard of $55 \mathrm{~dB}$ for a non work place. At a distance of 5 metres away from the generator, the total noise level is $96.6 \mathrm{dBA}$ which is above WHO standard of $85 \mathrm{~dB}$ in a work place. This level of noise should not be endured beyond the duration of 2 hours per day [3]. The equivalent continuous noise level of this generator at a distance of 5 metres is greater than that of $300 \mathrm{kVA}$ generators at the same distance by $4.6 \mathrm{dBA}$. The results of the finding show that the noise level when the generator was in operation is $99.6 \mathrm{dBA}$ at a distance of 5 metres away from it. This level is 14.6 $\mathrm{dBA}$ above the WHO standard level of $85 \mathrm{~dB}$ in a work place and $44.6 \mathrm{dBA}$ above the safety level of $55 \mathrm{~dB}$ for a non work place. The adverse effects of the generator noise are extended to a distance of 90 metres away from it. At this distance a total noise level of $56.7 \mathrm{dBA}$ instead of the WHO standard of $55 \mathrm{~dB}$ for a non work environment is recorded. Here, the residents from about 95 metres away from the generator, in the direction of the measurements do not have the generator as one of the major sources of noise. The results of the finding (Table 3.3 and Fig. 3.3) show that the total noise level with generator in use, the generator noise level and the equivalent continuous noise level decrease as the distance increases. The results (Table 3.4 and Fig. 3.4) of the findings show that at distances of 5 metres a total noise level of $89.7 \mathrm{dBA}$ is recorded. Out of the $89.7 \mathrm{dBA}, 53.8$ $\mathrm{dBA}$ comes from the workshop while $35.9 \mathrm{dBA}$ comes from the background. During the survey, it was observed that this workshop affects the residents seriously as its generators are located at distances of less than 5 metres away from some houses. Its adverse effects are extended to distances of about 60 metres where a total noise level of $55.5 \mathrm{dBA}$ is recorded. From a distance of about 65 metres, the workshop may not necessarily be the significant noise source to the residents. The results of the study (Table 3.5 and Fig. 3.5) show that the noise 
emanating from the workshop when this power generator is put to use and other noise makers adversely affect the residents up to distances of 60 metres with a total noise level of $56.7 \mathrm{dBA}$ which is $1.7 \mathrm{dBA}$ above the safety level of $55 \mathrm{dBA}$ for a non-work environment. Also the results show that the workshop is not a major source of noise from distances of about 65 metres away. With the simultaneous operation of three generators and other noise 'makers' the results show that the noise pollution of UPTECH Metal Construction Work extends to distances beyond 100 metres. This is because at this distance, the total noise level is $59.9 \mathrm{dBA}$ which is $4.4 \mathrm{dBA}$ beyond the WHO standard of $55 \mathrm{~dB}$ for a non-work place. Fig. 3.6 also shows that noise levels of the UPTECH Metal Construction Work decrease as distance from the noise sources increases. The results of the noise survey (Table 3.7 and Fig. 3.7) have revealed that the generator noise of this company adversely affects the residents up to a distance of 35 metres which has a total noise level of $2.5 \mathrm{dBA}$ higher than the WHO standard of $55 \mathrm{~dB}$ for a non-work environment. Here the impact of the noise is not much. From a distance of about 40 metres, the generator noise is less significant. Clearly, the results show that the generator sound level is $0 \mathrm{dBA}$ at a distance of 70 metres. This implies that beyond this distance the generator sound has no adverse effects on the populace. Also, the results of the findings show that sound level decreases as distance increases.

\section{Conclusion}

It is concluded from the survey that the equivalent continuous noise level, $\mathrm{L}_{\mathrm{eq}}$ which is a measure of energy content of the noise decreases as the distance from the noise source increases, hence degree of bother (or annoyance) decreases with distance.

\section{Acknowledgement}

We wish to thank the 2010 industrial training students from Akwa Ibom Broadcasting Corporation who assisted during the field works.

\section{References}

[1] A. I. Menkiti, Factors that Constitute Road Traffic Noise in Nigerian Environment, Global Journal of Pure and Applied Sciences, 7(3), 2001, 589.

[2] G. Kiely, Environmental engineering (Singapore, Irwin/McGraw-Hill Publishing Company Limited, 1998)

[3] FEPA, Guidelines to Standards for Environmental Pollution Control in Nigeria. Federal Environmental Protection Agency (FEPA), Lagos, 1991.

[4] E.E.C. Damage and Annoyance Caused by Noise EUR 5398e CEC Luxemburg, 1978

[5] Commission of the European Communities, Council Directive on the protection of workers from the risks related to exposure to noise at work', 86/18/EEC,1986.

[6] H.Ising, W. Babisch,, B. Kruppa, Noise-induced Edocrine Effects and Cardiovascular Risk, Noise Health,, 1(4), 1999, 37 - 48.

[7] A. A. Essiett, R.E. Akpan and S.O. Uwak, Assessment of Noise Level in Ikot Ekpene Town, Nigeria,. International Journal of Biotechnology and Allied Sciences, 5(1), 2010, 620 - 624.

[8] M.L.Davis, and D.A.Cornwell, Introduction to environmental engineering (New York, McGraw-Hill, 1991).

[9] OECD, Environmental Effects of Automotive Transport. The OECD Compass Project, OECD, Paris, 1986.

[10] WHO Guidelines for Community Noise, Transport Noise and Health: Transport and Environment, 2000, www.transportenvironment.org/module... Accessed Dec. 3, 2010.

[11] EC . The State of the Environment in the European Community, Publications of the European Communities, Luxemburg, 1986. 